\title{
Qualità del liquido seminale nella popolazione generale nei paesi Baltici
}

\author{
Alberto Ferlin 1
}

Pubblicato online: 11 luglio 2017

C S Springer International Publishing AG 2017

\section{Commento a:}

Semen quality of young men from the general population in Baltic countries.

J. Erenpreiss, M. Punab, B. Zilaitiene, S. Hlevicka, P. Zayakin, V. Matulevicius, R. Tomas Preiksa, N. Jørgensen. Hum Reprod (2017) 32(6):1334-1340

Accanto a una generale denatalità e aumento della infertilità di coppia, attribuibile ovviamente anche a cause non mediche, negli ultimi anni diverse segnalazioni e meta-analisi hanno documentato un declino temporale riferito agli ultimi decenni della qualità del liquido seminale. Anche alcuni studi trasversali hanno evidenziato un incremento della percentuale di alterazioni del liquido seminale negli uomini nati negli ultimi decenni. In generale, anche i valori di riferimento dei parametri seminali sono di pari passo diminuiti (vedi manuale del WHO per l'analisi del liquido seminale del 2010) e diverse segnalazioni indicano come una percentuale non trascurabile di giovani adulti abbiano valori di liquido seminale al di sotto di tali parametri, rientrando pertanto nella fascia dei soggetti con ridotti fertilità o a maggior rischio di infertilità. Esistono, tuttavia, differenze tra $\mathrm{i}$ diversi paesi e differenze anche regionali nella qualità del liquido seminale, probabilmente legate a influenze ambientali e diversi background genetici. Per esempio, tra i paesi del Nord Europa, i soggetti di Estonia, Lettonia, Lituania e Finlandia hanno parametri seminali migliori rispetto a quelli di Danimarca e Norvegia.

In questo studio sono stati valutati 1.165 giovani (età media 19,8 anni) della popolazione generale di Estonia (sia di

$\triangle$ A. Ferlin

alberto.ferlin@unipd.it

1 Dipartimento di Medicina, UOC Andrologia e Medicina della Riproduzione, Università di Padova, Padova, Italia etnia estone che russa), Lituania e Lettonia, reclutati su base volontaria (percentuali di adesione tra il 13 e il 17\%), mediante esame obiettivo, esame del liquido seminale e dosaggio di FSH e inibina B. Nel complesso, la mediana della concentrazione di spermatozoi era $63 \mathrm{milioni} / \mathrm{mL}$ e la mediana del numero totale di spermatozoi era 208 milioni/eiaculato, con differenze significative tra i diversi paesi e le etnie. Per esempio, tale parametro in Estonia di etnia estone era di 235 milioni/eiaculato, in Estonia di etnia russa era di 208 milioni/eiaculato. Anche il volume testicolare era significativamente diverso nello stesso senso. Di notevole interesse, al di là delle differenze tra i paesi e le etnie, è che una percentuale considerevole (11-15\%) degli uomini ha una bassa "qualità" del liquido seminale (utilizzando i cut-off di $<15$ milioni di spermatozoi/mL, $<32 \%$ di motilità progressiva e $<4 \%$ di morfologia normale). Tale percentuale è del $25-34 \%$ se si considera, però, il dato più importante del liquido seminale, cioè il numero totale di spermatozoi $<39$ milioni $/ \mathrm{mL}$.

Tale studio, pertanto, conferma alcuni dati estremamente interessanti, anche paragonati ad altri studi condotti in paesi europei (soprattutto Germania e Danimarca). La qualità del liquido seminale e, quindi, la funzionalità testicolare e dell'apparato riproduttivo nel complesso, presenta differenze sia a causa dell'influenza etnica (background genetico) che dell'influenza ambientale e degli stili di vita. Notevolmente preoccupa, però, il dato che circa un terzo dei giovani adulti è a rischio di infertilità o subfertilità. La loro funzione testicolare dovrebbe essere, pertanto, monitorata per prevenire o ridurre i danni di un ulteriore peggioramento e progressione del danno testicolare, fino all'età in cui tali soggetti decideranno di diventare genitori. Lo studio sottolinea, ancora una volta se ce ne fosse bisogno, l'importanza della prevenzione in campo andrologico e dello screening andrologico rivolto ai giovani. 